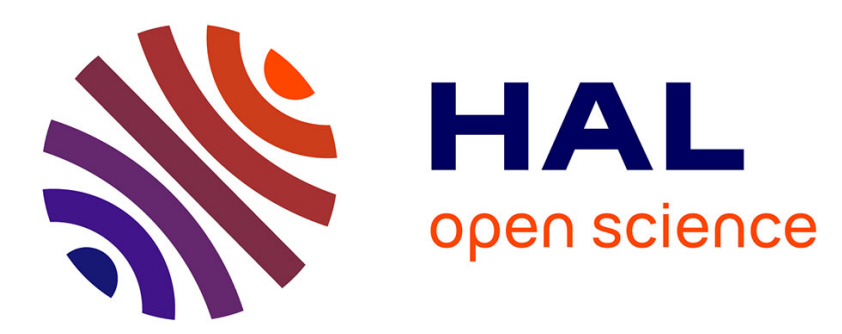

\title{
Bisexual branching processes to model extinction conditions for Y-linked genes
}

Miguel González, Rodrigo Martínez, Manuel Mota

\section{To cite this version:}

Miguel González, Rodrigo Martínez, Manuel Mota. Bisexual branching processes to model extinction conditions for Y-linked genes. Journal of Theoretical Biology, 2009, 258 (3), pp.478. 10.1016/j.jtbi.2008.10.034 . hal-00554526

\section{HAL Id: hal-00554526 \\ https://hal.science/hal-00554526}

Submitted on 11 Jan 2011

HAL is a multi-disciplinary open access archive for the deposit and dissemination of scientific research documents, whether they are published or not. The documents may come from teaching and research institutions in France or abroad, or from public or private research centers.
L'archive ouverte pluridisciplinaire HAL, est destinée au dépôt et à la diffusion de documents scientifiques de niveau recherche, publiés ou non, émanant des établissements d'enseignement et de recherche français ou étrangers, des laboratoires publics ou privés. 


\section{Author's Accepted Manuscript}

Bisexual branching processes to model extinction conditions for Y-linked genes

Miguel González, Rodrigo Martínez, Manuel Mota

PII: $\quad$ S0022-5193(08)00573-0

DOI: $\quad$ doi:10.1016/j.jtbi.2008.10.034

Reference: $\quad$ YJTBI5349

To appear in: $\quad$ Journal of Theoretical Biology

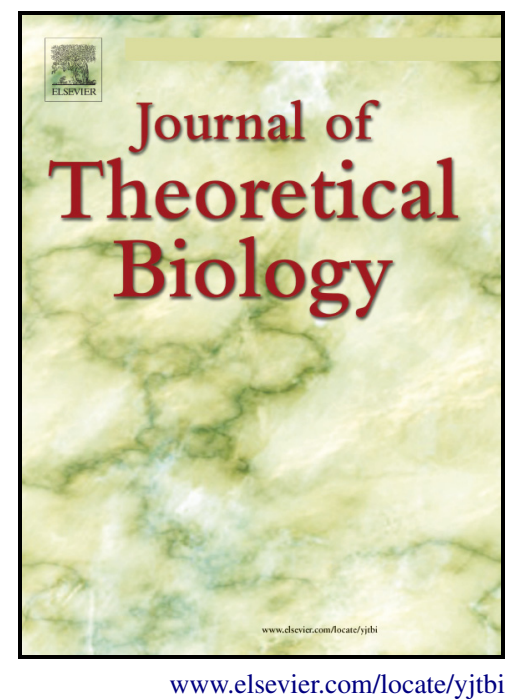

Received date: $\quad 30$ January 2008

Revised date: $\quad 27$ October 2008

Accepted date: 27 October 2008

Cite this article as: Miguel González, Rodrigo Martínez and Manuel Mota, Bisexual branching processes to model extinction conditions for Y-linked genes, Journal of Theoretical Biology (2008), doi:10.1016/j.jtbi.2008.10.034

This is a PDF file of an unedited manuscript that has been accepted for publication. As a service to our customers we are providing this early version of the manuscript. The manuscript will undergo copyediting, typesetting, and review of the resulting galley proof before it is published in its final citable form. Please note that during the production process errors may be discovered which could affect the content, and all legal disclaimers that apply to the journal pertain. 


\title{
Bisexual branching processes to model extinction conditions for Y-linked genes ${ }^{\star}$
}

\author{
Miguel González Rodrigo Martínez ${ }^{\star \star}$ Manuel Mota \\ Department of Mathematics, University of Extremadura, Badajoz 06071, Spain.
}

\begin{abstract}
In a two-sex monogamic population, the evolution of the number of carriers of the two alleles of a Y-linked gene is considered. To this end, a multitype bisexual branching model is presented in which it is assumed that the gene has no influence on the mating process. It is deduced from this model that the average numbers of female and male descendants per mating unit constitute the key to determining the extinction or survival of each allele. Moreover, the destiny of each allele in the population is found not to depend on the behaviour of the other.
\end{abstract}

Key words: Sex-linked inheritance, Y chromosome, two-dimensional bisexual stochastic model, perfect fidelity mating, survival.

\section{Introduction}

Sex determination in most mammal populations, including humans, is due to a pair of chromosomes denominated X and Y. Females have XX chromosomes, while males have two distinct chromosomes, XY. The SRY gene on the Y chromosome determines the development of the testes, and hence maleness (see Wallis et al. (2008)). The Y chromosome has very few genes in comparison with the $\mathrm{X}$ chromosome, but recent investigations have shown the importance of some Y-linked genes in populations of humans (see, for example, the web page www.nature.com/nature/focus/ychromosome/, Quintana-Murci and Fellous (2001), or Hughes et al. (2005)) and other animals which have the XX/XY

\footnotetext{
* The research was supported by the Ministerio de Educación y Ciencia and the FEDER through the Plan Nacional de Investigación Científica, Desarrollo e Innovación Tecnológica, grant MTM2006-08891.
}

${ }^{\star \star}$ Corresponding author. e-mail: rmartinez@unex.es 
type of sex determination system (see, for example, Bernardo et al. (2001), Gutiérrez and Teem (2006), or the review by Charlesworth et al. (2005)). Moreover this chromosome has the particularity of being male-specific and haploid, and of having a region (the non-recombining region, NRY, being $95 \%$ of the chromosome in humans - see, for example, Krausz et al. (2004) or Graves (2006)) which escapes recombination. These unique properties of the Y chromosome have important consequences for its population genetics: the NRY region passes down from father to son largely unchanged, and is therefore very useful for studying how populations have evolved. By examining the differences between modern Y chromosomes (such as DNA polymorphisms), one can attempt to reconstruct a history of paternal lineages. There have been many studies in this sense in the context of populations of humans (e.g., Hurles et al. (1998), Quintana-Murci et al. (2001), Hurles et al. (2002), or Rosa et al. (2007)) and other species (e.g., Tosi et al. (2002), Hellborg et al. (2005), or Geraldes et al. (2005)).

Another singular question associated with the $\mathrm{Y}$ chromosome is that of the microdeletions of this chromosome's long arm (Yq). These Yq deletions define three regions collectively known as AZF (azoospermia factors), with deletions of the AZFc region being the commonest. The Yq deletion is associated with males with fertility problems (for a review, see Krausz et al. (2003)), but many cases have been reported in which the natural transmission of this genetic defect from fathers to sons has occurred (see e.g., Saut et al. (2002), Calogero et al. (2002) or Kuhnert et al. (2004)). Related to this question, Krausz and McElreavey (2001) state: "The pathogenetic significance of Y chromosome microdeletions is spermatogenic failure and not infertility. AZF deletions can be associated with oligozoospermia which usually leads to infertility but it can also be associated with normal couple fertility. For the interpretation of any new observations about the transmission of Yq deletions we should keep in mind that fathering one or more children is the expression of the fertility status of both members of the couple". Obviously, determining the evolution of the number of males with this genetic defect in a human population is an important medical problem (see, for example, Patsalis et al. (2002) or Fitch et al. (2005)), but it has also been investigated in other species (e.g., Toure et al. (2004)).

The surname is another characteristic which can be seen as Y-linked in humans. There have been some recent studies aimed at determining the relationship between surnames and Y-chromosome lineages (e.g., King et al. (2006) or Bowden et al. (2008)).

Other organisms have a mirror image of the XX/XY sex determination system, with males being homogametic and females heterogametic. To avoid confusion, these sex chromosomes are deniminated $\mathrm{Z}$ and $\mathrm{W}$. Thus in birds, snakes, and butterflies, for example, females have ZW sex chromosomes, and males have ZZ 
sex chromosomes. Now, Y-linked problems become W-linked (see, for example, Ogawa et al. (1998), Yamada et al. (2004), or Abe et al. (2008)). For simplicity in notation, throughout this paper only the XX/XY sex determination system will be referred to, although the development and the results are equally valid for the ZZ/ZW system.

Appropriate mathematical models are needed to understand the evolution of $\mathrm{Y}$ chromosome lineages (for example, to help solve the problem of $\mathrm{Y}$ chromosomal Adam), Yq deletions, or other Y-linked genes.

Branching processes naturally come to mind in this context. These are stochastic models which arise in the description of population dynamics, being of particular use in describing the extinction/growth of populations (see Haccou et al. (2005)). Branching models have been applied to many biological problems in such fields as epidemiology, genetics, and cell dynamics. Examples include the evolution of infectious diseases (e.g., Mode and Sleemam (2000), Ball et al. (2004), or Garske and Rhodes (2008)), population genetics (e.g., Campbell (2003) or Iwasa et al. (2005)), and stem cells (e.g., Yakovlev and Yanev (2006)). Further examples are reviewed in the recent monographs of Kimmel and Axelrod (2002) and Pakes (2003), and in the communication of Caron-Lormier et al. (2006).

The simplest branching models, the Galton-Watson process and the Markov branching process, have been used to model Y-chromosome lineages and their female analogues - mitochondrial DNA lineages (see O'Conell (1995) and Neves and Moreira (2006)). But more accurate models are needed in which all the phases of sexual reproduction can be considered, including the interaction between females and males in producing offspring. In González et al. (2006), a model was presented that describes the evolution of the number of carriers of the two alleles of a Y-linked gene in a two-sex monogamic population. In that paper, it was considered that the characters controlled by such a gene can have some influence on the mating process of the species, with females having a preference for males carrying one of the alleles of the gene. It was shown that this preference can sometimes be definitive in determining the survival of the different genotypes in the population.

However, females' choice of mate is not always conditioned by the males' genotype. Most Y-linked characters do not appear in the phenotype of the males, or, even if they do, are not decisive at the time of mating (for example, in the case of Yq deletions or many of the NRY region genes). In these situations it seems more realistic to consider a model where females choose their mates without caring about what their genotype is, i.e., each female makes a blind choice of the genotype of her mate.

In this paper, we present a multitype bisexual branching process to model the 
evolution of the number of carriers of each allele of a Y-linked gene or of $\mathrm{Y}$ chromosome lineages in a two-sex monogamic population, assuming that this gene or characteristic does not influence the mating process. We focus our attention on studying the possibility of fixation of one of the alleles of the gene, and on determining the destiny of the gene in the population.

The reset of the paper consists of four sections. In Section 2 we provide the definition of the Y-linked Bisexual Branching Process assuming that females choose the genotypes of their mates blindly. We consider Y-linked genes with two alleles. We also present some basic properties of the model. In Section 3 we study the extinction problem for both the whole population and each genotype separately, in the latter case taking into account the behaviour of the other genotype. For some critical situations, we simulate the evolution of the population and conjecture its long term behaviour. In Section 4 we provide some concluding remarks. Finally, Section 5 presents the proofs of the results.

\section{The model}

Consider a human or other animal diploid population with non-overlapping generations, where the sex of an individual is determined by a pair of chromosomes $\mathrm{X}$ and $\mathrm{Y}$. A female $(\mathrm{F})$ will have $\mathrm{XX}$ chromosomes, while a male $(\mathrm{M})$ will have XY chromosomes.

Consider also a gene with a pair of alleles, $\mathrm{R}$ and $\mathrm{r}$, linked to the $\mathrm{Y}$ chromosome. The character defined by this gene is exclusive to males, which will be designated by MR or Mr according to which allele they carry. We will suppose however that they both have the same phenotype, so that mating is not influenced by the gene and females choose the genotype of their mates blindly. Moreover, perfect fidelity (monogamy) is assumed at the time of mating, i.e., each individual mates with only one individual of the opposite sex, provided that some of them are still available. Monogamy is the mating option in some birds and mammals, and in particular in most human populations, and is therefore well suited to the specific problems which motivated the present work (e.g., Yq deletions, or the history of paternal lineages).

A couple will be classified as FMR or FMr according to the genotype of the male. Following the inheritance rules, FMR mating units can generate females and MR males, while FMr mating units can produce females and Mr males.

We shall model the evolution of such a population by means of a two-type bisexual branching process in discrete time. To define this model, assume that it is known how many couples there are in the present generation, labeled in the definition as generation $n$. The objective is to determine the number 
of females, males, and mating units of each genotype in the next generation, labeled $n+1$. To this end, two phases will be considered: reproduction and mating.

In the reproduction phase, each couple of generation $n$ is assumed to produce offspring independently of the other couples according to a random variable. The probability distribution of these variables will be the same for all the couples with a given genotype, irrespective of the generation they belong to, and will be termed the reproduction law of that genotype. In general, FMR and FMr couples are assumed to have differences in their reproductive capacities, and consequently their respective reproduction laws are assumed to be different. This is an appropriate assumption in such problems as Yq deletions associated with male fertility problems. The important parameters will be the average numbers of offspring produced by FMR and FMr couples, denoted here by $m_{R}$ and $m_{r}$, respectively.

With probability $\alpha$ an offspring will be a female, and consequently with probability $1-\alpha$ it will be a male. It is assumed that the genotype has no influence on the sex determination, so that $\alpha$ is the same for both genotypes. Therefore, the average number of females and males per FMR couple will be $\alpha m_{R}$ and $(1-\alpha) m_{R}$, respectively, and these values will be $\alpha m_{r}$ and $(1-\alpha) m_{r}$, respectively, for an FMr couple.

The female offspring of all the couples in generation $n$ gives the total number of females in generation $n+1$, which will be denoted by $F_{n+1}$. On the basis of the genetic rules described above, the male offspring of all the FMR couples in generation $n$ gives the total number of MR males in generation $n+1$, denoted by $M R_{n+1}$. Analogously, the male offspring of all the FMr couples in generation $n$ gives the total number of Mr males in generation $n+1$, denoted by $M r_{n+1}$.

In the mating phase, the number of couples of each genotype formed in generation $n+1$ is calculated, taking into account that generations do not overlap and provided that in the population there are $F_{n+1}$ females, $M R_{n+1}$ males with $\mathrm{R}$ genotype, and $M r_{n+1}$ males with $\mathrm{r}$ genotype. We will denote by $Z R_{n+1}$ and $Z r_{n+1}$ the total number of FMR and FMr couples, respectively, in generation $n+1$.

The point here is the perfect fidelity mating considered in the premises of the model. According to this assumption, the total number of couples in generation $n+1$ is the minimum of the number of females, $F_{n+1}$, and the total number of males, $M R_{n+1}+M r_{n+1}$.

If the number of females exceeds that of males, then all the males mate, and consequently the number of couples of a genotype will be equal to the number of males of that genotype, i.e., $Z R_{n+1}=M R_{n+1}$ and $Z r_{n+1}=M r_{n+1}$. On the 
contrary, if the number of females is less than or equal to the number of males, all the females mate and must choose the genotype of their mates. However, since all the males have the same phenotype, each of the $F_{n+1}$ females makes a blind choice between the $M R_{n+1}$ males with $\mathrm{R}$ genotype and the $M r_{n+1}$ males with $\mathrm{r}$ genotype. Thus, the number of $F M R$ couples, $Z R_{n+1}$, will be given by a hypergeometric distribution of parameters $F_{n+1}, M R_{n+1}+M r_{n+1}$, and $M R_{n+1}$, i.e. $F_{n+1}$ males are selected from all males of generation $n+1$ where $M R_{n+1}$ males have the $\mathrm{R}$ genotype. The rest of the couples will have $\mathrm{r}$ genotype, i.e., $Z r_{n+1}=F_{n+1}-Z R_{n+1}$.

Obviously, the symmetry in the mating process means that it would be equivalent to consider $Z r_{n+1}$ given by a hypergeometric distribution of parameters $F_{n+1}, M R_{n+1}+M r_{n+1}$, and $M r_{n+1}$, and the number of remaining couples up to $F_{n+1}$ would be $Z R_{n+1}$. This symmetry is the main difference between the present model and that with preference in the mating introduced in González et al. (2006). In that model, the genetic character controlled by the gene does appear in the phenotype, and females prefer males carrying one of the alleles of the gene. The consequent lack of symmetry in the mating had a great influence on the results.

The bivariate sequence $\left\{\left(Z R_{n}, Z r_{n}\right)\right\}_{n \geq 0}$ indicating the generation-by-generation evolution of the number of mating units will be termed a Y-linked bisexual branching process.

From the definition of the model, the number of couples of each genotype in the next generation depends only on the present number of mating units, and not on the number of ancestors that belonged to past generations. Moreover, since each reproduction law remains the same over the generations, the transitions from one generation to another are homogeneous, i.e., they do not depend on the generation. The process $\left\{\left(Z R_{n}, Z r_{n}\right)\right\}_{n \geq 0}$ is therefore a homogeneous twotype Markov chain. However, the general theory of Markov chains, based on transition probabilities, does not lead to any significant results for this model. It is therefore necessary to attempt a specific methodological approach that is typical of branching processes.

The model introduced here shares some basic properties with the model with mating preference presented in González et al. (2006). These are properties which do not depend on the way the females choose their mates, and were proved in that paper. The following are four of these properties which are germane to the present work:

P 1 If in some generation there are no mating units of a particular genotype then, from this generation on, the mating units and the males of that genotype no longer exist. This is known as extinction of this particular genotype which implies the fixation of the allele determining the other genotype. The 
extinction of the whole population occurs when there are no mating units of any genotypes.

P 2 Provided that there exist couples of both genotypes in the present, there is a positive probability of reaching any number of mating units of each genotype in future generations. In particular, the fixation of one of the genotypes or even the extinction of the whole population are possible.

P 3 Each genotype presents the dual behaviour typical of branching processes: either it becomes extinct or the number of couples of this genotype eventually reaches arbitrarily large values. The latter event is known as explosion or indefinite growth of this particular genotype. Consequently the whole population also presents this duality. Thus, the survival of each genotype or of the whole population is equivalent to their indefinite growth as generations go by, with the possibility that, in the long term, their size tends to be in the neighbourhood of one or more positive values having to be discarded. Although this property might seem unrealistic, it merely expresses what would be the ideal long term evolution of a population when its development is not constrained by any external bound.

P 4 If the fixation of a particular allele has occurred, the corresponding genotype evolves essentially as a Bisexual Branching Process (BBP) with perfect fidelity mating and the reproduction law of the surviving genotype. Analogously, if both genotypes have the same reproduction law, the evolution of the total number of couples can also be modeled by a BBP with perfect fidelity mating.

With respect to this last property, it has to be remarked that the present model in some way inherits some of the properties of the BBP, a model that has been extensively investigated in the literature on Branching Processes. One may cite as examples work on the extinction probability (e.g., Daley (1968), Hull (1982, 1984), Bruss (1984), Daley et al. (1986), or Alsmeyer and Rösler (2002)), the long term behaviour (e.g., Bagley (1986) or González and Molina (1996, 1997)), and inference problems (e.g., González-Fragoso (1995), Molina et al. (1998), Alsmeyer and Rösler (1996), or González et al. (2001)). Two interesting recent reviews are Hull (2003) and Haccou et al. (2005).

Before concluding this section, it is worth comparing the Y-linked bisexual branching process with the classical genetic model of Wright-Fisher with which it shares some common features. In particular, both are Markov chains and consider diploid populations with non-overlapping generations. However there are also many differences. The most important is that, in the Wright-Fisher setting, the population size remains constant over the generations, reflecting a regulation of population size by external resources. The Wright-Fisher model may therefore not be the best model for a natural population. The Y-linked 
bisexual model, however, does allow indefinite growth. With respect to the mating, the Wright-Fisher model considers this to be random in the sense that every allele in a generation could come from every possible couple in the previous one owning that allele. In the Y-linked bisexual process, the number of couples owning each of the alleles, established in the mating phase, is of prime importance in determining the number of carriers of that allele in the subsequent generation.

\section{The extinction problem}

In this section we deal with two problems of interest in both the genetics and the population dynamics frameworks (e.g., Yq deletions, or the history of paternal lineages). The first is to obtain a necessary and sufficient condition for the whole population to become extinct almost surely. The other is to analyze the destiny of the gene in the population. To this end, we study some relevant events, such as the extinction of each genotype (mathematically, $\left\{Z R_{n} \rightarrow 0\right\}$ and $\left.\left\{Z r_{n} \rightarrow 0\right\}\right)$ and the survival or indefinite growth of each genotype (mathematically, $\left\{Z R_{n} \rightarrow \infty\right\}$ and $\left\{Z r_{n} \rightarrow \infty\right\}$ ). Survival is possible in the presence or absence of the other genotype, so we also consider the events $\left\{Z R_{n} \rightarrow \infty, Z r_{n} \rightarrow 0\right\}$ (fixation of $\mathrm{R}$ genotype), $\left\{Z R_{n} \rightarrow 0, Z r_{n} \rightarrow\right.$ $\infty\}$ (fixation of $\mathrm{r}$ genotype), and $\left\{Z R_{n} \rightarrow \infty, Z r_{n} \rightarrow \infty\right\}$ (survival of both genotypes), which are also of interest in themselves. We provide conditions depending on the parameters $m_{R}, m_{r}$, and $\alpha$.

We will assume that at the time the population was first observed, i.e., in generation 0 , there were $i$ couples with $\mathrm{R}$ genotype and $j$ couples with $\mathrm{r}$ genotype. In order to simplify the notation, we denote $P\left(\cdot \mid\left(Z R_{0}, Z r_{0}\right)=(i, j)\right)$ by $P_{(i, j)}(\cdot)$. Even $(i, j)$ will be dropped in this notation if there is no ambiguity.

\section{The extinction of the population}

It is understood that the population becomes extinct if from some generation on there are no mating units. For every $(i, j)$ we denote the probability of extinction of a population starting with $i$ FMR and $j$ FMr mating units by $Q_{(i, j)}=P_{(i, j)}\left(Z R_{n} \rightarrow 0, Z r_{n} \rightarrow 0\right)$.

Let $Q_{i}^{R}$ (resp. $Q_{j}^{r}$ ) be the probability of extinction of a BBP with perfect fidelity mating, reproduction law of $\mathrm{R}$ (resp. r) genotype, and $i$ (resp. $j$ ) starting mating units. From property $\mathrm{P} 4$, it follows that, for all $i, j \geq 0$,

$$
Q_{(i, 0)}=Q_{i}^{R} \text { and } Q_{(0, j)}=Q_{j}^{r},
$$


and therefore the results on the extinction of a BBP with perfect fidelity mating (see Daley (1968)) apply here.

When $i, j>0$, the following result provides a satisfactory answer to the problem of extinction.

Result 1 Let $i, j>0$. Then $Q_{(i, j)}=1$ if and only if $\min \left\{\alpha m_{r},(1-\alpha) m_{r}\right\} \leq 1$ and $\min \left\{\alpha m_{R},(1-\alpha) m_{R}\right\} \leq 1$.

We conclude that if the average number of females or males produced by a mating unit of each type is less than or equal to one then the process becomes extinct almost surely. On the other hand, if at least one of the values $\min \left\{\alpha m_{R},(1-\alpha) m_{R}\right\}$ or $\min \left\{\alpha m_{r},(1-\alpha) m_{r}\right\}$ is greater than one then, applying the results of a BBP, the corresponding genotype has a positive probability of survival even without any contribution of the other genotype, and therefore the population may survive too.

The destiny of the gene in the population

After determining a necessary and sufficient condition for the process to become extinct with probability one, we focus our interest on the long term growth of the number of carriers of each allele.

However, it would seem logical for there to exist some interdependence between the behaviour of the two genotypes, so that, in the analysis of each genotype, we must distinguish between whether the other has also an indefinite growth or has become extinct. In particular, we have to deal with the events

$\left\{Z R_{n} \rightarrow \infty, Z r_{n} \rightarrow 0\right\},\left\{Z R_{n} \rightarrow 0, Z r_{n} \rightarrow \infty\right\}$, and $\left\{Z R_{n} \rightarrow \infty, Z r_{n} \rightarrow \infty\right\}$

which represent the possible destinies of the two alleles in the population.

First we investigate the extinction of one genotype and the survival of the other. Because of the symmetry in the role of the two genotypes, the study of the fixation of each of them, $\left\{Z R_{n} \rightarrow \infty, Z r_{n} \rightarrow 0\right\}$ and $\left\{Z R_{n} \rightarrow 0, Z r_{n} \rightarrow\right.$ $\infty\}$, leads to analogous conclusions. In both events, the surviving genotype behaves from some generation on as a BBP with perfect fidelity mating (see property P4). Hence, the theory of Daley (1968) about a BBP applies, and it is immediate to deduce the following result.

Result 2 Let $i, j>0$, then

(i) $P_{(i, j)}\left(Z R_{n} \rightarrow \infty, Z r_{n} \rightarrow 0\right)>0$ if and only if $\min \left\{\alpha m_{R},(1-\alpha) m_{R}\right\}>1$.

(ii) $P_{(i, j)}\left(Z R_{n} \rightarrow 0, Z r_{n} \rightarrow \infty\right)>0$ if and only if $\min \left\{\alpha m_{r},(1-\alpha) m_{r}\right\}>1$. 
Intuitively, this result states that a necessary and sufficient condition for a genotype to have a positive probability of fixation is that both the female and the male descendants per couple of such a genotype are on average greater than one.

From the "if" parts of the above result, it is immediate to deduce the following corollary relative to the growth of the number of carriers of each allele.

Result 3 Let $i, j>0$, then

(i) If $\min \left\{\alpha m_{R},(1-\alpha) m_{R}\right\}>1$, then $P_{(i, j)}\left(Z R_{n} \rightarrow \infty\right)>0$.

(ii) If $\min \left\{\alpha m_{r},(1-\alpha) m_{r}\right\}>1$, then $P_{(i, j)}\left(Z r_{n} \rightarrow \infty\right)>0$.

With respect to the simultaneous survival of both genotypes, i.e., the event $\left\{Z R_{n} \rightarrow \infty, Z r_{n} \rightarrow \infty\right\}$, one has the following result.

Result 4 Let $i, j>0$.

(i) If $(1-\alpha) m_{R} \leq 1$ or $(1-\alpha) m_{r} \leq 1$, then $P_{(i, j)}\left(Z R_{n} \rightarrow \infty, Z r_{n} \rightarrow \infty\right)=0$.

(ii) If $\alpha m_{R}<1$ or $\alpha m_{r}<1$, then $P_{(i, j)}\left(Z R_{n} \rightarrow \infty, Z r_{n} \rightarrow \infty\right)=0$.

In Result 4 (i) we state that if the average number of male descendants per couple of either genotype is less than or equal to one, then there is null probability of the simultaneous survival of both genotypes. The key to this behaviour lies in the fact that the number of mating units of a genotype is bounded above by the number of males of that genotype.

Also, the probability of simultaneous survival of both genotypes is zero when the average number of female descendants per couple of either genotype is less than one. This is true, as indicated in Result 4 (ii), even though it could be expected that the males of this genotype would mate with females generated by mating units of the other genotype. For example, if one takes $\alpha<0.5, \alpha m_{R}<$ $1,(1-\alpha) m_{R}>1$, and $\alpha m_{r}>1$, even though the number of FMr mating units can grow indefinitely and the average number of MR male descendants per FMR mating unit is greater than one, the FMR mating units become extinct almost surely because not enough females are produced for all the population. This is an amazing fact, which in no way can be considered intuitive. To illustrate this scenario, we simulated 12 generations with $\left(Z R_{0}, Z r_{0}\right)=(50,1)$, $\alpha=0.3$, and the reproduction laws following Poisson distributions with $m_{R}=$ 2 and $m_{r}=4$. In Figure 1, we show a path of this process in which one observes the pattern described above.

The cases $\alpha m_{R}=1$ and $\alpha m_{r}=1$ are not considered in Result 4 (ii). If $\alpha \geq 0.5$, and $\alpha m_{R}=1$ or $\alpha m_{r}=1$, then $(1-\alpha) m_{R} \leq 1$ or $(1-\alpha) m_{r} \leq 1$, respectively, and Result 4 (i) guarantees that $P_{(i, j)}\left(Z R_{n} \rightarrow \infty, Z r_{n} \rightarrow \infty\right)=0$. However, if $\alpha<0.5$, we have not proved whether there is positive or null probability 

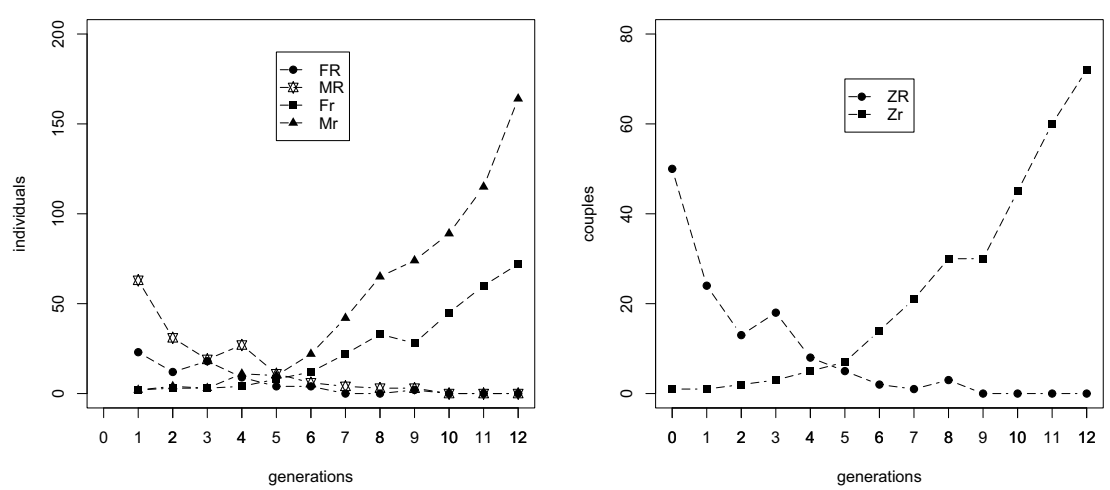

Fig. 1. Path of a process in which $\alpha m_{R}<1,(1-\alpha) m_{R}>1$, and $\alpha m_{r}>1$.

of the simultaneous survival of both genotypes. In order to conjecture the possible behaviour of the process in such situations, we performed a Monte Carlo simulation of ten batches of 10000 processes until generation 1000, with reproduction laws following Poisson distributions with means $m_{R}=2.5$ and $m_{r}=2.52$, and probability for an offspring to be female $\alpha=0.4$. Under these conditions $\alpha m_{R}=1,(1-\alpha) m_{R}=1.5>1$, and $\alpha m_{r}=1.008>1$. In all the simulated processes we took $\left(Z R_{0}, Z r_{0}\right)=(3,3)$. Table 1 lists the results for the number of processes in each batch where both genotypes have survived by a given generation. One observes how the number of such processes decreases to zero over the course of succeeding generations, with similar records for the ten batches. Also, Figure 2 shows the proportion of processes among the 100000 simulated ones in which both genotypes have survived by a given generation. Actually, this plot provides an estimate of the probability that both genotypes survive until generation $n$, with $n=1,2, \ldots$ Figure 3 shows the path of one process where both genotypes have survived until generation 1000. One observes geometric growth of the number of FMr mating units over the course of succeeding generations. The evolution of the number of FMR mating units, however, does not seem to fit any clear pattern, showing many fluctuations. However, according to property P3, it must converge either to 0 or $\infty$. Since this path does not seem to have an indefinite growth, we guess that eventually this path will become extinct, although it will take a great number of generations as can be observed in the figure. We could therefore conjecture that, in the situations with $\alpha m_{R}=1$ or $\alpha m_{r}=1$ not covered by Result 4, $P_{(i, j)}\left(Z R_{n} \rightarrow \infty, Z r_{n} \rightarrow \infty\right)=0$.

Applying property P3, it is immediate to deduce the following corollary of Results 2 and 4, related to the extinction of each allele.

Result 5 Let $i, j>0$, then

(i) If $\min \left\{\alpha m_{R},(1-\alpha) m_{R}\right\}<1$, then $P_{(i, j)}\left(Z R_{n} \rightarrow \infty\right)=0$. 


\begin{tabular}{l|cccccccccc} 
batch & 1 & 2 & 3 & 4 & 5 & 6 & 7 & 8 & 9 & 10 \\
\hline generation 20 & 233 & 242 & 221 & 223 & 219 & 245 & 199 & 223 & 229 & 239 \\
generation 40 & 55 & 58 & 49 & 54 & 45 & 48 & 49 & 51 & 56 & 49 \\
generation 60 & 27 & 29 & 24 & 25 & 18 & 22 & 29 & 30 & 27 & 27 \\
generation 80 & 18 & 17 & 15 & 17 & 11 & 13 & 14 & 21 & 14 & 17 \\
generation 100 & 13 & 12 & 11 & 15 & 7 & 12 & 11 & 15 & 12 & 13 \\
generation 200 & 3 & 3 & 2 & 4 & 2 & 4 & 4 & 7 & 6 & 6 \\
generation 300 & 3 & 2 & 1 & 1 & 2 & 4 & 4 & 3 & 4 & 5 \\
generation 400 & 3 & 2 & 1 & 1 & 1 & 1 & 1 & 2 & 4 & 2 \\
generation 500 & 3 & 2 & 0 & 0 & 1 & 1 & 1 & 2 & 4 & 2 \\
generation 1000 & 3 & 1 & 0 & 0 & 0 & 0 & 1 & 1 & 3 & 1
\end{tabular}

Table 1

Records of the number of processes in each batch where both genotypes have survived over the course of succeeding generations.

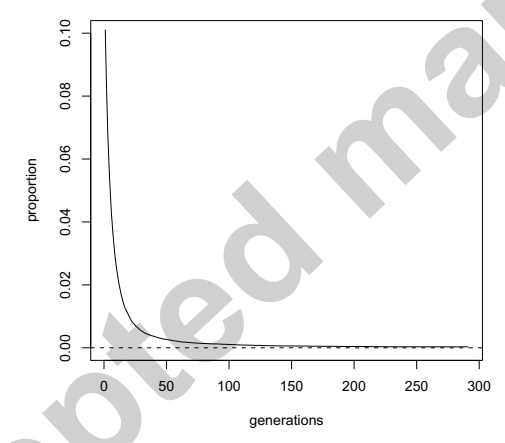

Fig. 2. Proportion over the course of succeeding generations of simulated processes in which both genotypes have survived.

(ii) If $\min \left\{\alpha m_{r},(1-\alpha) m_{r}\right\}<1$, then $P_{(i, j)}\left(Z r_{n} \rightarrow \infty\right)=0$.

Remark 1 From Results 3 and 5, one can infer that, roughly speaking, the extinction or survival of each genotype depends only on its own reproductive abilities, without any influence of the other genotype.

Finally, our interest now is to find conditions guaranteeing a positive probability of the simultaneous survival of both alleles. Such conditions are established in the following result.

Result 6 Let $i, j>0$. If $\alpha \neq 0.5, \min \left\{\alpha m_{R},(1-\alpha) m_{R}\right\}>1$ and $\min \left\{\alpha m_{r}\right.$, (1$\left.\alpha) m_{r}\right\}>1$, then $P_{(i, j)}\left(Z R_{n} \rightarrow \infty, Z r_{n} \rightarrow \infty\right)>0$. 

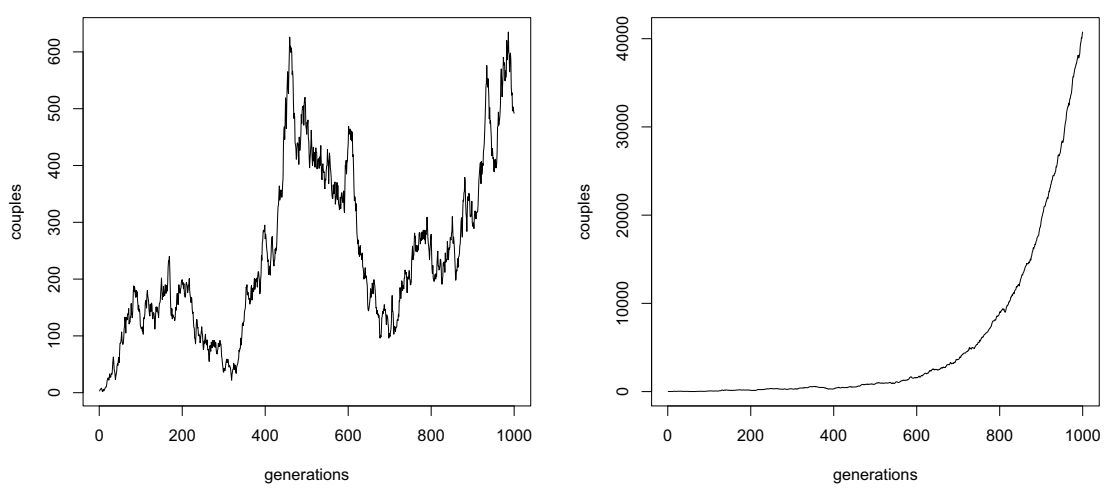

Fig. 3. Paths of the FMR (left) and FMr (right) genotypes in a process where both have survived until generation 1000 .

Intuitively, this result states that, for the simultaneous survival of both genotypes, the average number of male and of female descendants of each of them must be greater than one. This happens even in the case of very unbalanced sizes of the two genotypes. Actually, when $\alpha>0.5$ (resp. $\alpha<0.5$ ) and the number of couples of each genotype grows, then the number of females (resp. males) produced by all the mating units of each genotype is almost surely greater than the number of males (resp. females) produced by these mating units. Hence, the number of mating units of each genotype is determined by the number of males (resp. females) given by all the mating units of their genotype. Therefore, as we noted in the previous remark, each genotype must develop by itself in order for there to be a positive probability of simultaneous survival. Notice that this probability has not to be equal to one, i.e., there could be some paths where both genotypes do not survive simultaneously due to random fluctuations, even being all the thresholds parameters greater than one.

When, however, $\alpha=0.5$, the probability for the number of females to exceed that of males and the probability of there being more males than females are the same. In order to form a conjecture as to the possible behaviour of the process in this situation, where $\alpha m_{R}=(1-\alpha) m_{R}$ and $\alpha m_{r}=(1-\alpha) m_{r}$, we took both quantities to be greater than 1, performing a Monte Carlo simulation of ten batches of 10000 processes until generation 200, with reproduction laws following Poisson distributions with means $m_{R}=2.10$ and $m_{r}=2.15$. In all the simulated processes, we took $\left(Z R_{0}, Z r_{0}\right)=(50,50)$. Table 2 lists the results for the number of processes in each batch where both genotypes have survived by a given generation. One observes how the number of such processes decreases slowly to become stable over the course of succeeding generations, with similar records for the ten batches. Also, Figure 4 shows the proportion of processes among the 100000 simulated ones in which both genotypes have survived by a given generation. Actually, this plot provides 


\begin{tabular}{l|cccccccccc} 
batch & 1 & 2 & 3 & 4 & 5 & 6 & 7 & 8 & 9 & 10 \\
\hline generation 20 & 9798 & 9809 & 9825 & 9819 & 9796 & 9803 & 9806 & 9796 & 9798 & 9809 \\
generation 40 & 8112 & 8164 & 8071 & 8121 & 8084 & 8083 & 8128 & 8056 & 8063 & 8138 \\
generation 60 & 6508 & 6452 & 6402 & 6516 & 6463 & 6450 & 6484 & 6408 & 6425 & 6482 \\
generation 80 & 5566 & 5545 & 5553 & 5586 & 5526 & 5589 & 5634 & 5527 & 5547 & 5607 \\
generation 100 & 5136 & 5150 & 5177 & 5151 & 5108 & 5183 & 5221 & 5084 & 5102 & 5203 \\
generation 120 & 4953 & 4952 & 5009 & 4981 & 4937 & 5003 & 5036 & 4884 & 4931 & 5007 \\
generation 140 & 4879 & 4866 & 4903 & 4895 & 4860 & 4919 & 4949 & 4808 & 4851 & 4932 \\
generation 160 & 4841 & 4832 & 4873 & 4863 & 4832 & 4882 & 4907 & 4776 & 4816 & 4894 \\
generation 180 & 4825 & 4818 & 4863 & 4846 & 4817 & 4869 & 4887 & 4763 & 4794 & 4888 \\
generation 200 & 4820 & 4815 & 4856 & 4837 & 4813 & 4860 & 4881 & 4757 & 4790 & 4883 \\
Table 2 & & & & & & & & & &
\end{tabular}

Table 2

Records of the number of processes in each batch where both genotypes have survived over the course of succeeding generations.

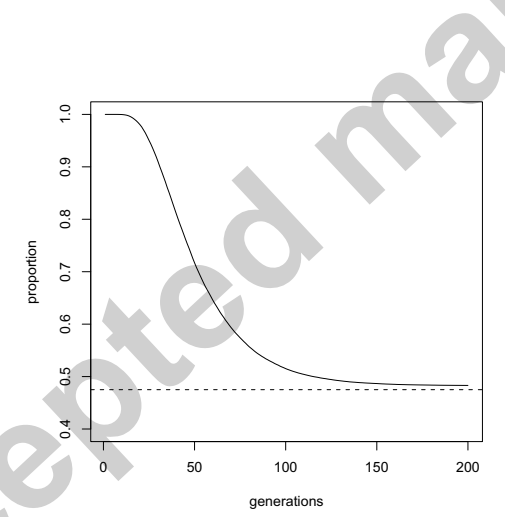

Fig. 4. Proportion over the course of succeeding generations of simulated processes in which both genotypes have survived.

an estimate of the probability that both genotypes survive until generation $n$, with $n=1,2, \ldots$ This probability is close to 0.475 for $n$ large enough. Figure 5 shows the logarithm of the number of couples of each genotype from the path of one process where both genotypes survive until generation 200. The observed linear behaviour leads one to deduce a geometric growth over the course of succeeding generations of the numbers of FMR and FMr mating units. We could therefore conjecture that, in the situations with $\alpha=0.5, \alpha m_{R}>1$, and $\alpha m_{r}>1$, not covered by Result $6, P_{(i, j)}\left(Z R_{n} \rightarrow \infty, Z r_{n} \rightarrow \infty\right)>0$. 

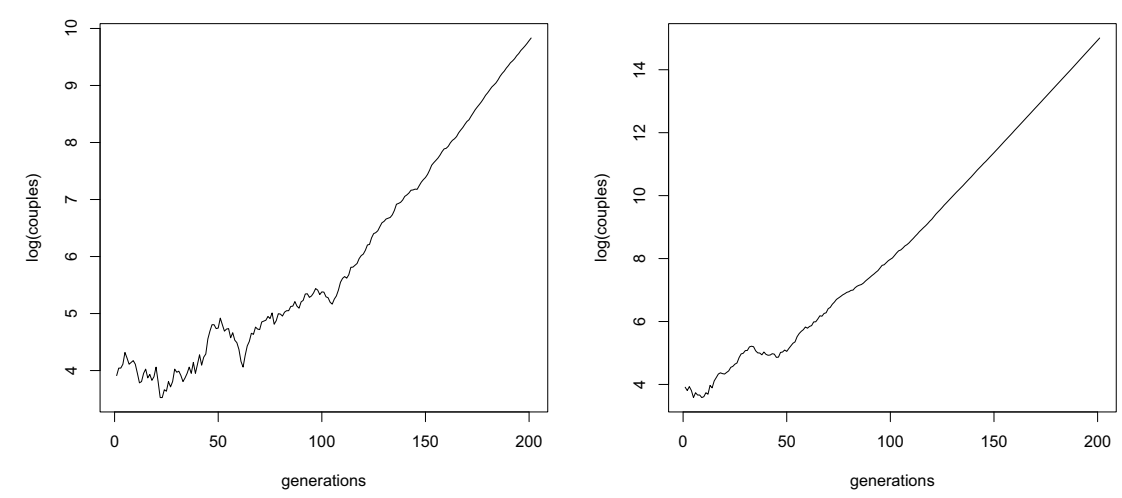

Fig. 5. Logarithm of the number of couples of the FMR (left) and FMr (right) genotypes from the path of one process where both genotypes have survived until generation 200, with $\alpha=0.5, \alpha m_{R}>1$, and $\alpha m_{r}>1$.

\section{Concluding remarks}

Two biological questions have been the motivation of this communication: Yq microdeletions leading to relative male infertility, and reconstruction of paternal lineages. Both questions were addressed as the transmission of a certain Y-linked gene or mark, with two alleles or versions, controlling characters which are not expressed in the individual's phenotype. These alleles could represent the presence or the absence of the character in individuals, such as having or not having Yq deletions or certain DNA polymorphisms.

We approached both situations by constructing a two-type bisexual branching process which models the generation-by-generation evolution of the number of carriers of such a Y-linked gene or mark in a two-sex monogamic population. In this framework, only males carry the gene and individuals (females or males) can mate with no more than one individual of the opposite sex in order to produce new individuals which will be females with a given probability (sexratio), the same for all generations. Moreover, we considered that all males have the same phenotype, and therefore a female chooses her mate blindly without caring about which genotype he has. As we pointed out in Section 2, monogamy is the predominant mating option in some birds and mammals, and in particular in most human populations, and is therefore well suited to the specific problems which motivated the present work (i.e., Yq deletions and the history of paternal lineages). But true monogamy, as considered in this paper, is difficult to find in nature. To relax this assumption, other mating options could have been considered, such as polygamous mating or population-sizedependent number of couples. It seems likely that these other types of mating would lead to different results, as indeed is the case in the monogamous mating model with preference of females for males carrying one of the alleles of the 
gene, studied in González et al. (2006), in relation to the simultaneous survival of the two genotypes.

Roughly speaking, we have shown that the extinction or survival of each genotype depends on the magnitudes of the average numbers of females and males produced by a mating unit of that genotype. Specifically, in the context of Yq deletions, this means that if the fertility rate of the couples formed by males with microdeletions is below a certain level, and there is no mutation, the males with this character will disappear from the population in the long term. On the contrary, if this fertility rate is above that level, there is a positive probability that the $\mathrm{Yq}$ deletions will remain in the population, even though their prevalence may be very low. This behaviour does not depend on the fertility rate of the couples formed by males without microdeletions. Therefore, although there is possible competition between the two types of males, this competition is not definitive for the presence of Yq deletions in the long term. Similar conclusions can be applied to the extinction or survival of paternal lineages. Indeed, this was the original problem which motivated work on branching processes (see Galton (1873)), although the first answers to this problem, based on the standard Galton-Watson branching model, did not take into account such biological parameters as mating option or sex ratio.

Moreover, our model has shown itself to have the advantage over the WrightFisher model in allowing fluctuations of the population size. Also, as pointed out above, it could be more useful than the Galton-Watson branching process for studies such as that of Neves and Moreira (2006) on Y-chromosomal Adam, because it considers a two-sex population and carefully models the mating process.

For a sex-ratio of 0.5 , some conclusions were established on the basis of simulations, but not analytically. Most populations in fact do not have a sex-ratio exactly equal to 0.5 , but slightly deviate from this value, and hence the analytical results of this paper apply. For a further development of this model, we could assume that the sex-ratio is not constant, but slightly biased according to the sex ratio in the population, with a tendency to move to compensate any deviation from the balance between the two sexes. In order to restore this balance, the probability for an offspring to be female in a generation could be taken, for example, as one minus the ratio between the number of females and the total number of individuals in the previous generation. This probability will be greater than 0.5 when there is an excess of males and less than 0.5 when there is an excess of females. As a result, we would obtain a populationsize-dependent reproduction law. This feature has already been included in branching model studies in both the asexual version (see, for example, Klebaner (1989)) and the bisexual version (see, for example, Xing and Wang (2005)). Following the lines of those studies, it would therefore be feasible to obtain analytical results under the conditions of such a scenario. 
Other questions for future mathematical research were also illustrated via simulation, such as the extinction time, for instance. In a simulated example, we showed that the time to extinction of a genotype can be extremely long when the other genotype grows indefinitely. The study of the probability distributions associated with these extinction times could be very relevant from a practical standpoint, for example in studying the problem of Y-chromosomal Adam. The rate of growth of a genotype given its non-extinction could also be an interesting problem to investigate. Another question for future research is to determine the probabilities of fixation of each genotype or of the simultaneous survival of both. While difficult, the analytical development of these questions may be feasible given the versatility of branching models in population dynamics modeling, and the many probabilistic tools available in the branching processes literature.

\section{Proofs}

First, we provide a formal definition of the Y-linked bisexual branching process. To this end, we consider two independent sequences $\left\{\left(F R_{n, l}, M R_{n, l}\right)\right.$ : $l=1,2, \ldots ; n=0,1, \ldots\}$ and $\left\{\left(F r_{n, l}, M r_{n, l}\right): l=1,2, \ldots ; n=0,1, \ldots\right\}$ of independent, identically distributed, non-negative, and integer-valued bivariate random vectors. Intuitively, $\left(F R_{n, l}, M R_{n, l}\right)$ (resp. $\left.\left(F r_{n, l}, M r_{n, l}\right)\right)$ represents the number of females and males generated by the $l$ th FMR (resp. FMr) mating unit in generation $n$.

With respect to the distribution of these reproduction vectors, we assume Daley's scheme (see Daley (1968)) with probability $\alpha(0<\alpha<1)$ for a descendant to be female. That is, the number of descendants of a FMR (resp. FMr) couple, $F R_{0,1}+M R_{0,1}$ (resp. $F r_{0,1}+M r_{0,1}$ ), is distributed according to the reproduction law $\left\{p_{k}^{R}\right\}_{k \geq 0}$ (resp. $\left.\left\{p_{k}^{r}\right\}_{k \geq 0}\right)$ with mean $m_{R}$ (resp. $m_{r}$ ) and probability generating function $f_{R}(\cdot)$ (resp. $f_{r}(\cdot)$ ). Then, since the probability of giving birth to a female is $\alpha$, the conditional distribution of $F R_{0,1}$ (resp. $F r_{0,1}$ ) given that $F R_{0,1}+M R_{0,1}=k$ (resp. $F r_{0,1}+M r_{0,1}=k$ ) is binomial of size $k$ and probability $\alpha$, and therefore $E\left[F R_{0,1}\right]=\alpha m_{R}$ and $E\left[M R_{0,1}\right]=$ $(1-\alpha) m_{R}$ (resp. $E\left[F r_{0,1}\right]=\alpha m_{r}$ and $\left.E\left[M r_{0,1}\right]=(1-\alpha) m_{r}\right)$.

Given the total number of FMR and FMr mating units at generation $n, Z R_{n}$ and $Z r_{n}$, respectively, the offspring generated by each genotype in generation $(n+1)$ is specified by the formulas

$$
\left(F R_{n+1}, M R_{n+1}\right)=\sum_{l=1}^{Z R_{n}}\left(F R_{n, l}, M R_{n, l}\right), \quad\left(F r_{n+1}, M r_{n+1}\right)=\sum_{l=1}^{Z r_{n}}\left(F r_{n, l}, M r_{n, l}\right),
$$

where the empty sum is assumed to be null. Intuitively, $\left(F R_{n+1}, M R_{n+1}\right)$ (resp. 
$\left.\left(F r_{n+1}, M r_{n+1}\right)\right)$ represents the total number of females and males, respectively, given by all the FMR (FMr) mating units in generation $n$. Moreover,

$$
F_{n+1}=F R_{n+1}+F r_{n+1}
$$

denotes the total number of females in generation $(n+1)$.

Given $\left(F_{n+1}, M R_{n+1}, M r_{n+1}\right)$, the total number of FMR and FMr couples in generation $n+1$ is given by the formulas

$$
Z R_{n+1}=M R_{n+1} \text { and } Z r_{n+1}=M r_{n+1}, \text { if } M R_{n+1}+M r_{n+1} \leq F_{n+1}
$$

and

$$
Z R_{n+1}=X_{n+1} \text { and } Z r_{n+1}=F_{n+1}-X_{n+1}, \text { if } M R_{n+1}+M r_{n+1}>F_{n+1}
$$

where $X_{n+1}$ follows a hypergeometric distribution of parameters $F_{n+1}, M R_{n+1}+$ $M r_{n+1}$, and $M R_{n+1}$ (see Hush and Scovel (2005) for details about the hypergeometric distribution).

Finally, we define the $\sigma$-algebras $\mathcal{F}_{n}=\sigma\left(\left(Z R_{k}, Z r_{k}\right): k=0, \ldots, n\right)$ and $\mathcal{G}_{n}=$ $\sigma\left(\left(Z R_{k}, Z r_{k}\right),\left(F R_{k+1}, M R_{k+1}\right),\left(F r_{k+1}, M r_{k+1}\right), k=0, \ldots, n\right), n=0,1, \ldots$

\section{Proof of Result 1}

The proof of this result follows the same ideas as that in Theorem 4.1 of González et al. (2006).

Proof of Result 4

We need the following lemma:

Lemma 2 Let $i, j>0$. If there exists a constant $A>0$ such that

$$
\begin{array}{ll} 
& E\left[Z R_{n+1} \mid\left(Z R_{n}, Z r_{n}\right)\right] \leq Z R_{n} \quad \text { a.s. on }\left\{Z R_{n} \geq A, Z r_{n} \geq A\right\} \\
\text { or } \quad & E\left[Z r_{n+1} \mid\left(Z R_{n}, Z r_{n}\right)\right] \leq Z r_{n} \text { a.s. on }\left\{Z R_{n} \geq A, Z r_{n} \geq A\right\},
\end{array}
$$

then $P_{(i, j)}\left(Z R_{n} \rightarrow \infty, Z r_{n} \rightarrow \infty\right)=0$.

Proof of the Lemma 
Given $A>0$ satisfying (1), it is enough to prove that for every $N>0$

$$
P_{(i, j)}\left(\left\{\inf _{n \geq N} Z R_{n} \geq A\right\} \cap\left\{\inf _{n \geq N} Z r_{n} \geq A\right\} \cap\left\{Z R_{n} \rightarrow \infty, Z r_{n} \rightarrow \infty\right\}\right)=0 .
$$

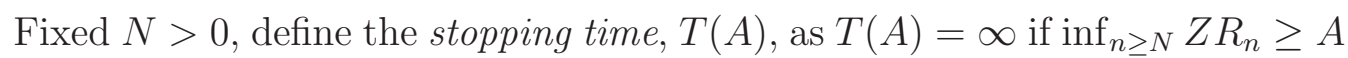
and $\inf _{n \geq N} Z r_{n} \geq A$, and $T(A)=\min \left\{n \geq N: Z R_{n}<A\right.$ or $\left.Z r_{n}<A\right\}$ otherwise.

Define also the sequence of random variables $\left\{Y_{n}\right\}_{n \geq 0}$ as follows:

$$
Y_{n}= \begin{cases}Z R_{N+n} & \text { if } N+n \leq T(A) \\ Z R_{T(A)} & \text { if } N+n>T(A) .\end{cases}
$$

To obtain (2), we show that $\left\{Y_{n}\right\}_{n \geq 0}$ is a non-negative supermartingale with respect to $\left\{\mathcal{F}_{N+n}\right\}_{n \geq 0}$. In fact, $Y_{n}$ is $\mathcal{F}_{N+n}$-measurable for any $n \geq 0$.

If $n \geq 0$ and $Z R_{N+k}, Z r_{N+k} \geq A, k=0, \ldots, n$, then $T(A) \geq N+n+1$, and from (1) we obtain that

$$
E\left[Y_{n+1} \mid \mathcal{F}_{N+n}\right]=E\left[Z R_{N+n+1} \mid \mathcal{F}_{N+n}\right] \leq Z R_{N+n}=Y_{n} \text { a.s. }
$$

on $\left\{Z R_{N+k}, Z r_{N+k} \geq A: k=0, \ldots, n\right\}$.

If $n \geq 1$ and for some $k \in\{1, \ldots, n\}$ it is satisfied that $Z R_{N}, \ldots, Z R_{N+k-1} \geq$ $A$ and $Z R_{N+k}<A$, or $Z r_{N}, \ldots, Z r_{N+k-1} \geq A$ and $Z r_{N+k}<A$, then $T(A) \leq$ $N+k<N+n+1$ and also

$$
E\left[Y_{n+1} \mid \mathcal{F}_{N+n}\right]=E\left[Z R_{T(A)} \mid \mathcal{F}_{N+n}\right]=Y_{n} \text { a.s. }
$$

on $\left\{Z R_{N}, \ldots, Z R_{N+k-1} \geq A, Z R_{N+k}<A\right\} \cup\left\{Z r_{N}, \ldots, Z r_{N+k-1} \geq A, Z r_{N+k}<\right.$ $A\}$. Finally, if $Z R_{N}<A$ or $Z r_{N}<A$ then $T(A)=N<N+n+1$ for all $n \geq 0$, and we get that

$$
E\left[Y_{n+1} \mid \mathcal{F}_{N+n}\right]=E\left[Z R_{N} \mid \mathcal{F}_{N+n}\right]=Y_{n} \text { a.s. on }\left\{Z R_{N}<A\right\} \cup\left\{Z r_{N}<A\right\} .
$$

In short, since $A_{n}=\left\{Z R_{N+k}, Z r_{N+k} \geq A, k=0, \ldots, n\right\} \in \mathcal{F}_{N+n}$, one deduces that

$$
\begin{aligned}
E\left[Y_{n+1} \mid \mathcal{F}_{N+n}\right] & =E\left[Y_{n+1} I_{A_{n}} \mid \mathcal{F}_{N+n}\right]+E\left[Y_{n+1} I_{A_{n}^{c}} \mid \mathcal{F}_{N+n}\right] \\
& =E\left[Y_{n+1} \mid \mathcal{F}_{N+n}\right] I_{A_{n}}+E\left[Y_{n+1} \mid \mathcal{F}_{N+n}\right] I_{A_{n}^{c}} \\
& \leq Z R_{N+n} I_{A_{n}}+Z R_{T(A)} I_{A_{n}^{c}}=Y_{n} \text { a.s. }
\end{aligned}
$$

Applying the Martingale Convergence Theorem, we obtain the almost sure convergence of the sequence $\left\{Y_{n}\right\}_{n \geq 0}$ to a non-negative and finite limit, $Y_{\infty}$, 
where

$$
Y_{\infty}= \begin{cases}\lim _{n \rightarrow \infty} Z R_{n} & \text { if } \inf _{n \geq N} Z R_{n} \geq A \text { and } \inf _{n \geq N} Z r_{n} \geq A \\ Z R_{T(A)} & \text { otherwise. }\end{cases}
$$

Therefore we deduce (2) and the proof of the Lemma is complete.

\section{Proof of the Result}

(i) Assume $(1-\alpha) m_{R} \leq 1$. The proof is analogous for $(1-\alpha) m_{r} \leq 1$ because of the symmetry of the model with respect to the two genotypes. Using the definition of the process,

$$
E\left[Z R_{n} \mid \mathcal{G}_{n-1}\right]=\left\{\begin{array}{ll}
M R_{n} & \text { if } F_{n} \geq M R_{n}+M r_{n} \\
M R_{n} \frac{F_{n}}{M R_{n}+M r_{n}} & \text { if } F_{n}<M R_{n}+M r_{n}
\end{array}\right. \text { a.s. }
$$

so that $E\left[Z R_{n} \mid \mathcal{G}_{n-1}\right] \leq M R_{n}$ a.s. and, since $\mathcal{F}_{n} \subseteq \mathcal{G}_{n}, n=0,1, \ldots$,

$$
\begin{aligned}
E\left[Z R_{n} \mid \mathcal{F}_{n-1}\right] & =E\left[E\left[Z R_{n} \mid \mathcal{G}_{n-1}\right] \mid \mathcal{F}_{n-1}\right] \leq E\left[M R_{n} \mid \mathcal{F}_{n-1}\right] \\
& =(1-\alpha) m_{R} Z R_{n-1} \leq Z R_{n-1} \quad \text { a.s. }
\end{aligned}
$$

Applying the previous Lemma, we obtain that $P\left(Z R_{n} \rightarrow \infty, Z r_{n} \rightarrow \infty\right)=0$.

(ii) Assume $\alpha m_{R}<1$. The proof is analogous for $\alpha m_{r}<1$. Assume also $\alpha<0.5$. Otherwise the result is deduced from (i).

According to the previous Lemma we have to show (1) for some constant $A$. But, using the definition of the process,

$$
\begin{aligned}
& E\left[Z R_{n} \mid \mathcal{G}_{n-1}\right]=M R_{n} I_{\left\{F_{n} \geq M R_{n}+M r_{n}\right\}}+\frac{M R_{n} F_{n}}{M R_{n}+M r_{n}} I_{\left\{F_{n}<M R_{n}+M r_{n}\right\}} \\
\leq & M R_{n} I_{\left\{F R_{n} \geq M R_{n}\right\}}+M R_{n} I_{\left\{F r_{n} \geq M r_{n}\right\}}+\frac{M R_{n} F_{n}}{M R_{n}+M r_{n}} I_{\left\{F_{n}<M R_{n}+M r_{n}\right\}} \text { a.s. }
\end{aligned}
$$

Let us bound properly the conditional expectation given $\mathcal{F}_{n-1}$ of each of these summands.

For the first summand of (3), since we are assuming Daley's scheme, we can apply the bounds for the tails of a binomial distribution given by Okamoto's inequality (see Johnson et al. (1993)), and we have, for all $n$ and $k>0$,

$$
\begin{aligned}
P\left(F R_{n} \geq M R_{n} \mid F R_{n}+M R_{n}=k\right) & =P\left(F R_{n} \geq k / 2 \mid F R_{n}+M R_{n}=k\right) \\
& \leq e^{-k(0.5-\alpha)^{2}}=a_{1}^{k},
\end{aligned}
$$


with $a_{1}=e^{-(0.5-\alpha)^{2}}<1$. Then, for all $i^{\prime}, j^{\prime}>0$

$$
\begin{aligned}
& P\left(F R_{n} \geq M R_{n} \mid Z R_{n-1}=i^{\prime}, Z r_{n-1}=j^{\prime}\right) \\
= & E\left[P\left(F R_{n} \geq M R_{n} \mid F R_{n}+M R_{n}\right) \mid Z R_{n-1}=i^{\prime}, Z r_{n-1}=j^{\prime}\right] \\
\leq & E\left[a_{1}^{F R_{n}+M R_{n}} \mid Z R_{n-1}=i^{\prime}, Z r_{n-1}=j^{\prime}\right]=f_{R}\left(a_{1}\right)^{i^{\prime}} .
\end{aligned}
$$

Therefore, applying (4) and the Cauchy-Schwartz inequality,

$$
\begin{aligned}
E\left[M R_{n} I_{\left\{F R_{n} \geq M R_{n}\right\}} \mid \mathcal{F}_{n-1}\right] & \leq E\left[M R_{n}^{2} \mid \mathcal{F}_{n-1}\right]^{1 / 2} P\left(F R_{n} \geq M R_{n} \mid \mathcal{F}_{n-1}\right)^{1 / 2} \\
& \leq K_{1} Z R_{n-1} f_{R}\left(a_{1}\right)^{Z R_{n-1} / 2} \quad \text { a.s. }
\end{aligned}
$$

for some positive constant $K_{1}$.

For the second summand of (3), we can proceed analogously and obtain

$$
E\left[M R_{n} I_{\left\{F r_{n} \geq M r_{n}\right\}} \mid \mathcal{F}_{n-1}\right] \leq K_{2} Z R_{n-1} f_{r}\left(a_{1}\right)^{Z r_{n-1} / 2} \quad \text { a.s. }
$$

for some positive constant $K_{2}$.

To bound the third summand of (3), given $\varepsilon>0$, define $\gamma_{1}=\alpha\left(m_{R}-\varepsilon\right)$, $\gamma_{2}=\alpha\left(m_{R}+\varepsilon\right), \gamma_{3}=(1-\alpha)\left(m_{R}-\varepsilon\right), \gamma_{4}=(1-\alpha)\left(m_{R}+\varepsilon\right), \gamma_{5}=\alpha\left(m_{r}-\varepsilon\right)$, $\gamma_{6}=\alpha\left(m_{r}+\varepsilon\right), \gamma_{7}=(1-\alpha)\left(m_{r}-\varepsilon\right), \gamma_{8}=(1-\alpha)\left(m_{r}+\varepsilon\right)$, and $B_{\varepsilon}=$ $1+2 \varepsilon /\left(\min \left\{m_{R}, m_{r}\right\}-\varepsilon\right)$. We take $\varepsilon$ such that $\gamma_{1}, \gamma_{5}>0$ and $0<\gamma_{2} B_{\varepsilon}<1$.

For each $n \geq 1$, define also

$$
\begin{aligned}
& A_{F R, n}=\left\{\left|F R_{n}-Z R_{n-1} \alpha m_{R}\right| \leq Z R_{n-1} \alpha \varepsilon\right\}, \\
& A_{M R, n}=\left\{\left|M R_{n}-Z R_{n-1}(1-\alpha) m_{R}\right| \leq Z R_{n-1}(1-\alpha) \varepsilon\right\}, \\
& A_{F r, n}=\left\{\left|F r_{n}-Z r_{n-1} \alpha m_{r}\right| \leq Z r_{n-1} \alpha \varepsilon\right\}, \\
& A_{M r, n}=\left\{\left|M r_{n}-Z r_{n-1}(1-\alpha) m_{r}\right| \leq Z r_{n-1}(1-\alpha) \varepsilon\right\} .
\end{aligned}
$$

From now on, $n$ will be dropped in the notation if there is no ambiguity.

Since the reproduction laws are assumed to have finite variances, an immediate application of Chebyshev's inequality gives

$$
P\left(A_{F R}^{c} \cup A_{M R}^{c} \mid Z R_{n-1}=i^{\prime}, Z r_{n-1}=j^{\prime}\right) \leq \frac{C_{1}}{i^{\prime}}
$$

and

$$
P\left(A_{F r}^{c} \cup A_{M r}^{c} \mid Z R_{n-1}=i^{\prime}, Z r_{n-1}=j^{\prime}\right) \leq \frac{C_{2}}{j^{\prime}}
$$

for some positive constants $C_{1}$ and $C_{2}$. 
Now, if we denote $D=A_{F R} \cap A_{M R} \cap A_{F r} \cap A_{M r}$,

$$
\begin{aligned}
& E\left[\frac{M R_{n} F_{n}}{M R_{n}+M r_{n}} I_{\left\{F_{n}<M R_{n}+M r_{n}\right\}} \mid \mathcal{F}_{n-1}\right] \\
\leq & E\left[\frac{M R_{n} F_{n}}{M R_{n}+M r_{n}} I_{\left\{F_{n}<M R_{n}+M r_{n}\right\}} I_{D} \mid \mathcal{F}_{n-1}\right] \\
& +E\left[\frac{M R_{n} F_{n}}{M R_{n}+M r_{n}} I_{\left\{F_{n}<M R_{n}+M r_{n}\right\}} I_{A_{F R}^{c} \cup A_{M R}^{c}} \mid \mathcal{F}_{n-1}\right] \\
& +E\left[\frac{M R_{n} F_{n}}{M R_{n}+M r_{n}} I_{\left\{F_{n}<M R_{n}+M r_{n}\right\}} I_{A_{F r}^{c} \cup A_{M r}^{c}} \mid \mathcal{F}_{n-1}\right] \text { a.s. }
\end{aligned}
$$

Applying (7) and the Cauchy-Schwartz inequality,

$$
\begin{aligned}
& E\left[\frac{M R_{n} F_{n}}{M R_{n}+M r_{n}} I_{\left\{F_{n}<M R_{n}+M r_{n}\right\}} I_{A_{F R}^{c} \cup A_{M R}^{c}} \mid \mathcal{F}_{n-1}\right] \leq E\left[M R_{n} I_{A_{F R}^{c} \cup A_{M R}^{c}} \mid \mathcal{F}_{n-1}\right] \\
\leq & E\left[M R_{n}^{2} \mid \mathcal{F}_{n-1}\right]^{1 / 2} P\left(A_{F R}^{c} \cup A_{M R}^{c} \mid \mathcal{F}_{n-1}\right)^{1 / 2} \\
\leq & \left(K_{3}^{\prime} Z R_{n-1}\right)\left(C_{1} Z R_{n-1}\right)^{-1 / 2}=K_{3} Z R_{n-1}^{1 / 2} \quad \text { a.s. }
\end{aligned}
$$

for some positive constants $K_{3}^{\prime}$ and $K_{3}$.

Analogously,

$$
E\left[\frac{M R_{n} F_{n}}{M R_{n}+M r_{n}} I_{\left\{F_{n}<M R_{n}+M r_{n}\right\}} I_{A_{F r}^{c} \cup A_{M r}^{c}} \mid \mathcal{F}_{n-1}\right] \leq K_{4} Z R_{n-1} Z r_{n-1}^{-1 / 2} \quad \text { a.s. }
$$

for some positive constant $K_{4}$.

Finally,

$$
\begin{aligned}
& E\left[\frac{M R_{n} F_{n}}{M R_{n}+M r_{n}} I_{\left\{\hat{F}_{n}<M R_{n}+M r_{n}\right\}} I_{D} \mid \mathcal{F}_{n-1}\right] \\
\leq & \gamma_{4} Z R_{n-1} \frac{\gamma_{2} Z R_{n-1}+\gamma_{6} Z r_{n-1}}{\gamma_{3} Z R_{n-1}+\gamma_{7} Z r_{n-1}} \\
= & \alpha\left(m_{R}+\varepsilon\right) Z R_{n-1} \frac{\left(m_{R}+\varepsilon\right) Z R_{n-1}+\left(m_{r}+\varepsilon\right) Z r_{n-1}}{\left(m_{R}-\varepsilon\right) Z R_{n-1}+\left(m_{r}-\varepsilon\right) Z r_{n-1}} \\
= & \gamma_{2} Z R_{n-1} \frac{\left(m_{R}-\varepsilon\right) Z R_{n-1}+2 \varepsilon Z R_{n-1}+\left(m_{r}-\varepsilon\right) Z r_{n-1}+2 \varepsilon Z r_{n-1}}{\left(m_{R}-\varepsilon\right) Z R_{n-1}+\left(m_{r}-\varepsilon\right) Z r_{n-1}} \\
\leq & \gamma_{2} Z R_{n-1}\left(1+\frac{2 \varepsilon}{\min \left\{m_{R}-\varepsilon, m_{r}-\varepsilon\right\}}\right)=\gamma_{2} B_{\varepsilon} Z R_{n-1} \quad \text { a.s. }
\end{aligned}
$$

Summarizing, from (5), (6), (9), (10), (11), and (12), we deduce that

$$
\begin{aligned}
E\left[Z R_{n} \mid \mathcal{F}_{n-1}\right] & \leq\left(K_{1} f_{R}\left(a_{1}\right)^{Z R_{n-1} / 2}+K_{2} f_{r}\left(a_{1}\right)^{Z r_{n-1} / 2}\right. \\
& \left.+K_{3} Z R_{n-1}^{-1 / 2}+K_{4} Z r_{n-1}^{-1 / 2}+\gamma_{2} B_{\varepsilon}\right) Z R_{n-1} \quad \text { a.s. }
\end{aligned}
$$


Since $a_{1}<1$ and $\gamma_{2} B_{\varepsilon}<1$, we can take $A>0$ such that, for $Z R_{n-1}>A$ and $Z r_{n-1}>A$, the term in parentheses is less than 1, so that (1) holds and therefore the result is proved.

\section{Proof of Result 6}

For each $\eta_{1}, \eta_{2}>1$, let $A_{n}=\left\{Z R_{n+1}>\eta_{1} Z R_{n}, Z r_{n+1}>\eta_{2} Z r_{n}\right\}$ for all $n \geq 0$

$$
\begin{aligned}
& P_{(i, j)}\left(Z R_{n} \rightarrow \infty, Z r_{n} \rightarrow \infty\right) \geq P_{(i, j)}\left(\bigcap_{n=0}^{\infty}\left\{Z R_{n+1}>\eta_{1} Z R_{n}, Z r_{n+1}>\eta_{2} Z r_{n}\right\}\right) \\
= & \lim _{n \rightarrow \infty} P_{(i, j)}\left(\bigcap_{l=0}^{n} A_{l}\right)=\lim _{n \rightarrow \infty} P_{(i, j)}\left(A_{0}\right) \prod_{l=1}^{n} P_{(i, j)}\left(A_{l} \mid \bigcap_{k=0}^{l-1} A_{k}\right) .
\end{aligned}
$$

Moreover, using the Markov property of $\left\{\left(Z R_{n}, Z r_{n}\right)\right\}_{n \geq 0}$, for all $n>0$

$$
\begin{aligned}
& P_{(i, j)}\left(A_{n} \mid \bigcap_{k=0}^{n-1} A_{k}\right)=P_{(i, j)}\left(A_{n} \mid \bigcup_{i^{\prime} j^{\prime}>0}\left\{\left(Z R_{n}, Z r_{n}\right)=\left(i^{\prime}, j^{\prime}\right)\right\} \cap \bigcap_{k=0}^{n-1} A_{k}\right) \\
\geq & \inf _{i^{\prime}>\eta_{1}^{n} i, j^{\prime}>\eta_{2}^{n} j} P_{(i, j)}\left(A_{n} \mid\left\{\left(Z R_{n}, Z r_{n}\right)=\left(i^{\prime}, j^{\prime}\right)\right\} \cap \bigcap_{k=0}^{n-1} A_{k}\right) \\
= & \inf _{i^{\prime}>\eta_{1}^{n} i, j^{\prime}>\eta_{2}^{n} j} P_{\left(i^{\prime}, j^{\prime}\right)}\left(A_{0}\right) .
\end{aligned}
$$

Let us bound conveniently $P_{\left(i^{\prime}, j^{\prime}\right)}\left(A_{0}\right)$ for all $i^{\prime}, j^{\prime}>0$ or equivalently $P_{\left(i^{\prime}, j^{\prime}\right)}\left(A_{0}^{c}\right)$. First we assume that $\alpha>0.5$, and both $(1-\alpha) m_{R}$ and $(1-\alpha) m_{r}$ are greater than 1 . Take $\varepsilon>0, \eta_{1}=(1-\alpha) m_{R}-\varepsilon$, and $\eta_{2}=(1-\alpha) m_{r}-\varepsilon$ such that $\eta_{1}, \eta_{2}>1$. Then

$$
\begin{aligned}
A_{0}^{c}= & \left\{Z R_{1} \leq \eta_{1} Z R_{0}\right\} \cup\left\{Z r_{1} \leq \eta_{2} Z r_{0}\right\} \\
\subseteq & \left\{Z R_{1} \leq \eta_{1} Z R_{0}, M R_{1}>\eta_{1} Z R_{0}, F R_{1}>M R_{1}, F r_{1}>M r_{1}\right\} \\
& \cup\left\{M R_{1} \leq \eta_{1} Z R_{0}\right\} \cup\left\{F R_{1} \leq M R_{1}\right\} \\
& \cup\left\{Z r_{1} \leq \eta_{2} Z r_{0}, M r_{1}>\eta_{2} Z r_{0}, F R_{1}>M R_{1}, F r_{1}>M r_{1}\right\} \\
& \cup\left\{M r_{1} \leq \eta_{2} Z r_{0}\right\} \cup\left\{F r_{1} \leq M r_{1}\right\} .
\end{aligned}
$$

Since $Z R_{1}=M R_{1}$ and $Z r_{1}=M r_{1}$ if $M R_{1}+M r_{1}<F_{1}$, then we have that

$$
P_{\left(i^{\prime}, j^{\prime}\right)}\left(Z R_{1} \leq \eta_{1} Z R_{0}, M R_{1}>\eta_{1} Z R_{0}, F R_{1}>M R_{1}, F r_{1}>M r_{1}\right)=0
$$

and

$$
P_{\left(i^{\prime}, j^{\prime}\right)}\left(Z r_{1} \leq \eta_{2} Z r_{0}, M r_{1}>\eta_{2} Z r_{0}, F R_{1}>M R_{1}, F r_{1}>M r_{1}\right)=0 .
$$


Moreover, since the reproduction laws are assumed to have finite variances, by Chebyshev's inequality, it follows that

$$
P_{\left(i^{\prime}, j^{\prime}\right)}\left(M R_{1} \leq \eta_{1} Z R_{0}\right)=P\left(\sum_{k=1}^{i^{\prime}}\left(M R_{k 0}-(1-\alpha) m_{R}\right) \leq-\varepsilon i^{\prime}\right) \leq \frac{C_{1}}{i^{\prime}}
$$

and analogously

$$
P_{\left(i^{\prime}, j^{\prime}\right)}\left(M r_{1} \leq \eta_{2} Z r_{0}\right) \leq \frac{C_{2}}{j^{\prime}},
$$

for some constants $C_{1}, C_{2}>0$.

In the same way as (4), we obtain, for some constant $a_{1}<1$, that

$$
P_{\left(i^{\prime}, j^{\prime}\right)}\left(F R_{1} \leq M R_{1}\right) \leq f_{R}\left(a_{1}\right)^{i^{\prime}} \text { and } P_{\left(i^{\prime}, j^{\prime}\right)}\left(F r_{1} \leq M r_{1}\right) \leq f_{r}\left(a_{1}\right)^{j^{\prime}} \text {. }
$$

From (15), (16), (17), (18), (19), and (20), we have

$$
P_{\left(i^{\prime}, j^{\prime}\right)}\left(A_{0}\right)=1-P_{\left(i^{\prime}, j^{\prime}\right)}\left(A_{0}^{c}\right) \geq 1-\frac{C_{1}}{i^{\prime}}-\frac{C_{2}}{j^{\prime}}-f_{R}\left(a_{1}\right)^{i^{\prime}}-f_{r}\left(a_{1}\right)^{j^{\prime}}
$$

and therefore, since $\eta_{1}, \eta_{2}>1$ and $f_{R}\left(a_{1}\right), f_{r}\left(a_{1}\right)<1$, from (13) and (14) it follows that

$$
\begin{aligned}
& P_{(i, j)}\left(Z R_{n} \rightarrow \infty, Z r_{n} \rightarrow \infty\right) \\
\geq & P_{(i, j)}\left(A_{0}\right) \lim _{n \rightarrow \infty} \prod_{l=1}^{n} \inf _{i^{\prime}>\eta_{1}^{l} i, j^{\prime}>\eta_{2}^{l} j} P_{\left(i^{\prime}, j^{\prime}\right)}\left(A_{0}\right) \\
\geq & P_{(i, j)}\left(A_{0}\right) \lim _{n \rightarrow \infty} \prod_{l=1}^{n}\left(1-\frac{C_{1}}{\eta_{1}^{l} i}-\frac{C_{2}}{\eta_{2}^{l} j}-f_{R}\left(a_{1}\right)^{\eta_{1}^{l} i}-f_{r}\left(a_{1}\right)^{\eta_{2}^{l} j}\right)>0,
\end{aligned}
$$

and the proof is complete for $\alpha>0.5$.

Assume now that $\alpha<0.5$, and both $\alpha m_{R}$ and $\alpha m_{r}$ are greater than 1 . Given $\varepsilon>0$, define the constants $\gamma_{1}, \ldots, \gamma_{8}$ and the sets $A_{F R}, A_{M R}, A_{F r}, A_{M r}$ and $D$ as in the proof of Result 4. Take $\varepsilon$ small enough so that $\gamma_{2}<\gamma_{3}, \gamma_{6}<\gamma_{7}$, and

$$
\gamma_{1}\left(1-\frac{2 \varepsilon}{\min \left\{m_{R}+\varepsilon, m_{r}+\varepsilon\right\}}\right)>1, \gamma_{5}\left(1-\frac{2 \varepsilon}{\min \left\{m_{R}+\varepsilon, m_{r}+\varepsilon\right\}}\right)>1
$$

and choose

$$
1<\eta_{1}<\gamma_{1}\left(1-\frac{2 \varepsilon}{\min \left\{m_{R}+\varepsilon, m_{r}+\varepsilon\right\}}\right)
$$


and

$$
1<\eta_{2}<\gamma_{5}\left(1-\frac{2 \varepsilon}{\min \left\{m_{R}+\varepsilon, m_{r}+\varepsilon\right\}}\right) .
$$

Specifically, we take $\eta_{1}=\gamma_{1}\left(1-3 \varepsilon / \min \left\{m_{R}+\varepsilon, m_{r}+\varepsilon\right\}\right)$ and $\eta_{2}=\gamma_{5}(1-$ $3 \varepsilon / \min \left\{m_{R}+\varepsilon, m_{r}+\varepsilon\right\}$.

With this notation

$$
A_{0}^{c} \subseteq\left(\left\{Z R_{1} \leq \eta_{1} Z R_{0}\right\} \cap D\right) \cup\left(\left\{Z r_{1} \leq \eta_{2} Z r_{0}\right\} \cap D\right) \cup D^{c} .
$$

Equations (7) and (8) provide a suitable bound for $P_{\left(i^{\prime}, j^{\prime}\right)}\left(D^{c}\right)$, so we focus our attention on the two first terms of (21).

Since $\gamma_{2}<\gamma_{3}$ and $\gamma_{6}<\gamma_{7}, F_{1}<M R_{1}+M r_{1}$ on $D$, and, by the definition of the process, the conditional distribution of $Z R_{1}$ given $\mathcal{G}_{0}=\sigma\left(F_{1}, M R_{1}, M r_{1}\right)$ is hypergeometric. Hence

$$
\begin{aligned}
& P_{\left(i^{\prime}, j^{\prime}\right)}\left(\left\{Z R_{1} \leq \eta_{1} Z R_{0}\right\} \cap D\right)=E_{\left(i^{\prime}, j^{\prime}\right)}\left[P_{\left(i^{\prime}, j^{\prime}\right)}\left(Z R_{1} \leq \eta_{1} Z R_{0} \mid \mathcal{G}_{0}\right) I_{D}\right] \\
= & E_{\left(i^{\prime}, j^{\prime}\right)}\left[P_{\left(i^{\prime}, j^{\prime}\right)}\left(Z R_{1}-E_{\left(i^{\prime}, j^{\prime}\right)}\left[Z R_{1} \mid \mathcal{G}_{0}\right] \leq \eta_{1} i^{\prime}-\frac{M R_{1} F_{1}}{M R_{1}+M r_{1}} \mid \mathcal{G}_{0}\right) I_{D}\right]
\end{aligned}
$$

But on $D$

$$
\begin{aligned}
\eta_{1} i^{\prime}-\frac{M R_{1} F_{1}}{M R_{1}+M r_{1}} & \leq \eta_{1} i^{\prime}-\gamma_{3} i^{\prime} \frac{\gamma_{1} i^{\prime}+\gamma_{5} j^{\prime}}{\gamma_{4} i^{\prime}+\gamma_{8} j^{\prime}} \\
& =\eta_{1} i^{\prime}-\gamma_{1} i^{\prime} \frac{\left(m_{R}-\varepsilon\right) i^{\prime}+\left(m_{r}-\varepsilon\right) j^{\prime}}{\left(m_{R}+\varepsilon\right) i^{\prime}+\left(m_{r}+\varepsilon\right) j^{\prime}} \\
& =\eta_{1} i^{\prime}-\gamma_{1} i^{\prime} \frac{\left(m_{R}+\varepsilon\right) i^{\prime}+\left(m_{r}+\varepsilon\right) j^{\prime}-2 \varepsilon\left(i^{\prime}+j^{\prime}\right)}{\left(m_{R}+\varepsilon\right) i^{\prime}+\left(m_{r}+\varepsilon\right) j^{\prime}} \\
& \leq\left[\eta_{1}-\gamma_{1}\left(1-\frac{2 \varepsilon}{\min \left\{m_{R}+\varepsilon, m_{r}+\varepsilon\right\}}\right)\right] i^{\prime} \\
& =-\frac{\varepsilon i^{\prime}}{\min \left\{m_{R}+\varepsilon, m_{r}+\varepsilon\right\}} \gamma_{1} .
\end{aligned}
$$

Let us write $\delta=\gamma_{1} \varepsilon / \min \left\{m_{R}+\varepsilon, m_{r}+\varepsilon\right\}$. Applying the previous inequality and the bounds for the tails of a hypergeometric distribution provided in Hush and Scovel (2005), for $i^{\prime}$ large enough, we can deduce from (22)

$$
\begin{aligned}
& P_{\left(i^{\prime}, j^{\prime}\right)}\left(\left\{Z R_{1} \leq \eta_{1} Z R_{0}\right\} \cap D\right) \\
\leq & E_{\left(i^{\prime}, j^{\prime}\right)}\left[P_{\left(i^{\prime}, j^{\prime}\right)}\left(Z R_{1}-E_{\left(i^{\prime}, j^{\prime}\right)}\left[Z R_{1} \mid \mathcal{G}_{0}\right] \leq-\delta i^{\prime} \mid \mathcal{G}_{0}\right) I_{D}\right] \\
\leq & E_{\left(i^{\prime}, j^{\prime}\right)}\left[\exp \left\{-2 \frac{\delta^{2} i^{\prime 2}-1}{M R_{n}+1}\right\} I_{D}\right] \leq \exp \left\{-2 \frac{\delta^{2} i^{\prime 2}-1}{\gamma_{4} i^{\prime}+1}\right\} \leq K_{1} e^{-B_{1} i^{\prime}}
\end{aligned}
$$

for some positive constants $K_{1}$ and $B_{1}$. Notice that, without loss of generality, we can take $i$, and consequently $i^{\prime}$, large enough because all the states with both coordinates non-null are communicating. 
Analogously, it can be deduced that

$$
P_{\left(i^{\prime}, j^{\prime}\right)}\left(\left\{Z r_{1} \leq \eta_{1} Z r_{0}\right\} \cap D\right) \leq K_{2} e^{-B_{2} j^{\prime}}
$$

for some positive constants $K_{2}$ and $B_{2}$.

Then, taking into account the decomposition in (21), from (7), (8), (23), and (24), we deduce that

$$
P_{\left(i^{\prime}, j^{\prime}\right)}\left(A_{0}\right)=1-P_{\left(i^{\prime}, j^{\prime}\right)}\left(A_{0}^{c}\right) \geq 1-K_{1} e^{-B_{1} i^{\prime}}-K_{2} e^{-B_{2} j^{\prime}}-\frac{C_{1}}{i^{\prime}}-\frac{C_{2}}{j^{\prime}},
$$

and therefore, since $\eta_{1}, \eta_{2}>1$ and $B_{1}, B_{2}>0$, from (13) and (14) it follows that

$$
\begin{aligned}
& P_{(i, j)}\left(Z R_{n} \rightarrow \infty, Z r_{n} \rightarrow \infty\right) \geq P_{(i, j)}\left(A_{0}\right) \lim _{n \rightarrow \infty} \prod_{l=1}^{n} \inf _{i^{\prime}>\eta_{1}^{l} i, j^{\prime}>\eta_{2}^{l} j} P_{\left(i^{\prime}, j^{\prime}\right)}\left(A_{0}\right) \\
& \geq P_{(i, j)}\left(A_{0}\right) \lim _{n \rightarrow \infty} \prod_{l=1}^{n}\left(1-K_{1} e^{-B_{1} \eta_{1}^{l} i}-K_{2} e^{-B_{2} \eta_{2}^{l} j}-\frac{C_{1}}{\eta_{1}^{l} i}-\frac{C_{2}}{\eta_{2}^{l} j}\right)>0,
\end{aligned}
$$

which completes the proof for $\alpha>0.5$.

\section{Acknowledgement}

We would like to thank the anonymous referees for their constructive comments and interesting suggestions which have improved this paper.

\section{References}

Abe, H., Fujii, T., Tanaka, N., Yokoyama, T., Kakehashi, H., Ajimura, M., Mita, K., Banno, Y., Yasukochi, Y., Oshiki, T., Nenoi, M., Ishikawa, T., Shimada, T., 2008. Identification of the female-determining region of the $\mathrm{W}$ chromosome in Bombyx mori. Genetica 133, 269-282.

Alsmeyer, G., Rösler, U., 1996. The bisexual Galton-Watson branching process with promiscuous mating: extinction probabilities in the supercritical case. Ann. Appl. Probab. 6, 922-939.

Alsmeyer, G., Rösler, U., 2002. Asexual versus promiscuous bisexual GaltonWatson branching processes: the extinction probability ratio. Ann. Appl. Probab. 12, 125-142.

Bagley, J. H., 1986. On the asymptotic properties of a supercritical bisexual branching process. J. Appl. Probab. 23, 820-826. 
Ball, F., Britton, T., Lyne, O., 2004. Stochastic multitype epidemics in a community of households: estimation of threshold parameter $\mathrm{R}^{*}$ and secure vaccination coverage. Biometrika 91(2), 345-362.

Bernardo, A., Dobo, B., Vibranovski, M., Clark, A., 2001. Identification of five new genes on the Y chromosome of Drosophila melanogaster. Proc. Natl. Acad. Sci. USA 98 (23), 13225-13230.

Bowden, G., Balaresque, P., King, T., Hansen, Z., Lee, A., Pergl-Wilson, G., Hurley, E., Roberts, S., Waite, P., Jesch, J., Jones, A., Thomas, M., Harding, S., Jobling, M., 2008. Excavating past population structures by surnamebased sampling: the genetic legacy of the Vikings in northwest England. Molecular Biology and Evolution 25 (2), 301-309.

Bruss, F. T., 1984. A note on extinction criteria for bisexual Galton-Watson processes. J. Appl. Probab. 21, 915-919.

Calogero, A., Garofalo, M., Barone, N., Longo, G., De Palma, A., Fichera, M., Rappazzo, G., D’Agata, R., Vicari, E., 2002. Spontaneous transmission from a father to his son of a Y chromosome microdeletion involving the deleted in azoospermia(DAZ) gene. J Endocrinol Invest 25, 631-634.

Campbell, R., 2003. A logistic branching process for population genetics. Journal of Theoretical Biology 225 (2), 195-203.

Caron-Lormier, G., Masson, J., Mnard, N., Pierre, J., 2006. A branching process, its application in biology: Influence of demographic parameters on the social structure in mammal groups. Journal of Theoretical Biology 238 (3), $564-574$.

Charlesworth, D., Charlesworth, B., Marais, G., 2005. Steps in the evolution of heteromorphic sex chromosomes. Heredity 95, 118-128.

Daley, D. J., Hull, D. M., Taylor, J. M., 1986. Bisexual Galton-Watson branching processes with superadditive mating functions. J. Appl. Probab. 23, 585-600.

Daley, D. J., 1968. Extinction conditions for certain bisexual Galton-Watson branching processes. Z. Wahrscheinlichkeitsth. 9, 315-322.

Fitch, N., Richer, C., Pinsky, L., Kahn, A., Opitz, J., Reynolds, J., 2005. Deletion of the long arm of the $\mathrm{Y}$ chromosome and review of $\mathrm{Y}$ chromosome abnormalities. American Journal of Medical Genetics 20 (1), 31-42.

Galton, F., 1873. Problem 4001: On the extinction of surnames. Educational Times 26, 1717.

Garske, T., Rhodes, C., 2008. The effect of superspreading on epidemic outbreak size distributions. Journal of Theoretical Biology 253 (2), 228-237.

Geraldes, A., Rogel-Gaillard, C., Ferrand, N., 2005. High levels of nucleotide diversity in the European rabbit (Oryctolagus cuniculus) SRY gene. Animal Genetics 36 (4), 349-351.

González-Fragoso, A., 1995. Ratio estimation for the offspring means of a bisexual Galton-Watson branching process. J. Inter-American Stat. Inst. 47, 17-36.

González, M., Hull, D. M., Martínez, R., Mota, M., 2006. Bisexual branching processes in a genetic context: The extinction problem for Y-linked genes. 
Math. Biosci. 202, 227-247.

González, M., Molina, M., Mota, M., 2001. Nonparametric estimation of the offspring distribution and the mean vector for a bisexual Galton-Watson process. Comm. Statist. Theory Methods 30, 497-516.

González, M., Molina, M., 1996. On the limit behaviour of a superadditive bisexual Galton-Watson branching process. J. Appl. Probab. 33, 960-967.

González, M., Molina, M., 1997. On the $L^{2}$-convergence of a superadditive bisexual Galton-Watson branching process. J. Appl. Probab. 34, 575-582.

Graves, J., 2006. Sex chromosome specialization and degeneration in mammals. Cell 124(5), 901-914.

Gutiérrez, J., Teem, J., 2006. A model describing the effect of sex-reversed YY fish in an established wild population: The use of a trojan Y chromosome to cause extinction of an introduced exotic species. Journal of Theoretical Biology 241, 333-341.

Haccou, P., Jagers, P., Vatutin, V., 2005. Branching processes: variation, growth and extinction of populations. Cambridge University Press.

Hellborg, L., Gndz, I., Jaarola, M., 2005. Analysis of sex-linked sequences supports a new mammal species in Europe. Molecular Ecology 14 (7), 20252031.

Hughes, J. F., Skaletsky, H., Pyntikova, T., Minx, P. J., Graves, T., Rozen, S., Wilson, R. K., Page, D. C., 2005. Conservation of Y-linked genes during human evolution revealed by comparative sequencing in chimpanzee. Nature 437, 100-103.

Hull, D. M., 1982. A necessary condition for extinction in those bisexual Galton-Watson branching processes governed by superadditive mating functions. J. Appl. Probab. 19, 847-850.

Hull, D. M., 1984. Conditions for extinction in certain bisexual Galton-Watson branching processes. J. Appl. Probab. 21, 414-418.

Hull, D. M., 2003. A survey of the literature associated with the bisexual Galton-Watson branching processes. Extracta Math. 13, 321-343.

Hurles, M., Irven, C., Nicholson, J., Taylor, P., Santos, F., Loughlin, J., Jobling, M., Sykes, B., 1998. European Y-chromosomal lineages in Polynesians: a contrast to the population structure revealed by mtDNA. Am J Hum Genet. 63 (6), 1793-1806.

Hurles, M., Nicholson, J., Bosch, E., Renfrew, C., Sykes, B., Jobling, M., 2002. Y chromosomal evidence for the origins of Oceanic-speaking peoples. Genetics 160 (1), 289-303.

Hush, D., Scovel, C., 2005. Concentration of the hypergeometric distribution. Statist. Probab. Lett. 75, 127-132.

Iwasa, Y., Michor, F., Komarova, N., Nowak, M., 2005. Population genetics of tumor suppressor genes. Journal of Theoretical Biology 233 (1), 15-23.

Johnson, N. L., Kemp, A. W., Kotz, S., 1993. Univariate discrete distributions. John Wiley and Sons, Inc.

Kimmel, M., Axelrod, D., 2002. Branching processes in biology. SpringerVerlag. 
King, T., Ballereau, S., Schrer, K., Jobling, M., 2006. Genetic signatures of coancestry within surnames. Curr. Biol. 16 (4), 384-388.

Klebaner, F., 1989. Geometric growth in near-supercritical population-sizedependent multitype Galton-Watson processes. Ann. Prob. 17, 14661477.

Krausz, C., Forti, G., McElreavey, K., 2003. The Y chromosome and male fertility and infertility. Int J Androl 26, 70-75.

Krausz, C., McElreavey, K., 2001. Y chromosome microdeletions in fertile males. Hum Reprod. 16 (6), 1306-1307.

Krausz, C., Quintana-Murci, L., Forti, G., 2004. Y chromosome polymorphisms in medicine. Ann Med. 36 (8), 573-583.

Kuhnert, B., Gromoll, J., Kostova, E., Tschanter, P., Luetjens, C., Simoni, M., Nieschlag, E., 2004. Case report: natural transmission of an AZFc Ychromosomal microdeletion from father to his sons. Hum Reprod. 19, 886 888.

Mode, C. J., Sleemam, C. K., 2000. Stochastic processes in epidemiology. World Scientific.

Molina, M., González, M., Mota, M., 1998. Bayesian inference for bisexual Galton-Watson processes. Comm. Statist. Theory Methods 27, 1055-1070.

Neves, A., Moreira, C., 2006. Applications of the GaltonWatson process to human DNA evolution and demography. Physica A 368, 132146.

O'Conell, N., 1995. The genealogy of branching processes and the age of our more recent common ancestor. Adv. Appl. Probab. 27, 418-442.

Ogawa, A., Murata, K., Mizuno, S., 1998. The location of Z- and W-linked marker genes and sequence on the homomorphic sex chromosomes of the ostrich and the emu. Proc. Natl. Acad. Sci. USA 95, 4415-4418.

Pakes, A., 2003. Biological applications of branching processes. Handbook of Statistic Vol. 21 Stochastic Processes: Modelling and Simulation (Shanbhag, D.N. and Rao, C.R., eds.) Chapter 18, 693-773, Elsevier Science B.V.

Patsalis, P., Sismani, C., Quintana-Murci, L., Taleb-Bekkouche, F., Krausz, C., McElreavey, K., 2002. Effects of transmission of Y chromosome AZFc deletions. The Lancet 360 (9341), 1222-1224.

Quintana-Murci, L., Fellous, M., 2001. The human Y chromosome: the biological role of a "functional wasteland". J. Biomed. Biotechnol. 1, 18-24.

Quintana-Murci, L., Krausz, C., Zerjal, T., Sayar, S. H., Hammer, M. F., Mehdi, S., Ayub, Q., Qamar, R., Mohyuddin, A., Radhakrishna, U., Jobling, M., Tyler-Smith, C., McElreavey, K., 2001. Y-chromosome lineages trace diffusion of people and languages in southwestern Asia. Am J Hum Genet. $68(2), 537-542$.

Rosa, A., Ornelas, C., Jobling, M., Brehm, A., Villems, R., 2007. Ychromosomal diversity in the population of Guinea-Bissau: a multiethnic perspective. BMC Evol Biol. 27, 107-124.

Saut, N., Terriou, P., Navarro, A., Lvy, N., Mitchell, M., 2002. The human Y chromosome genes BPY2, CDY1 and DAZ are not essential for sustained fertility. Mol. Hum. Reprod. 6, 789-793.

Tosi, A., Morales, J., Melnick, D., 2002. Y-chromosome and mitochondrial 
markers in Macaca fascicularis indicate introgression with Indochinese M. mulatta and a biogeographic barrier in the Isthmus of Kra. Int. J. Primatol. $23(1), 161-178$.

Toure, A., Szot, M., Mahadevaiah, S., Rattigan, A., Ojarikre, O., Burgoyne, P., 2004. A new deletion of the mouse Y chromosome long arm associated with the loss of Ssty expression, abnormal sperm development and sterility. Genetics 166, 901-912.

Wallis, M., Waters, P., Graves, J., 2008. Sex determination in mammals before and after the evolution of SRY. Cell. Mol. Life Sci. to appear. doi:10.1007/s00018-008-8109-z.

Xing, Y., Wang, Y., 2005. On the extinction of a class of population-sizedependent bisexual branching processes. J. Appl. Probab. 42 (1), 175184.

Yakovlev, A., Yanev, N., 2006. Branching stochastic processes with immigration in analysis of renewing cell populations. Math. Biosci 203, 37-63.

Yamada, D., Koyama, Y., Komatsubara, M., Urabe, M., Mori, M., Hashimoto, Y., Nii, R., Kobayashi, M., Nakamoto, A., Ogihara, J., Kato, J., Mizuno, S., 2004. Comprehensive search for chicken W chromosome-linked genes expressed in early female embryos from the female-minus-male subtracted cDNA macroarray. Chromosome Research, 12, 741-754. 